\title{
Module partition for mechanical CAD assembly model based on multi-source correlation information and community detection
}

\author{
Zhoupeng HAN*, Rong MO*, Haicheng YANG* and Li HAO* \\ *Key Laboratory of Contemporary Design and Integrated Manufacturing Technology, Ministry of Education, \\ Northwestern Polytechnical University, Xi'an 710072, China \\ E-mail: hanzhoupeng@126.com
}

Received: 10 July 2017; Revised: 26 November 2017; Accepted: 1 February 2018

\begin{abstract}
To solve the problem of difficulty in mechanical CAD assembly model retrieval and low level of the model reuse, provide effective support for module reuse in assembly model, a novel module partition method for mechanical CAD assembly model is proposed. Firstly, the correlation strength between assembled parts is analyzed and evaluated based on multi-source correlation information including assembly structure, function and flows. Then, the weighted network is constructed for expressing correlation relationships between assembled parts in the assembly model. After that, a community detection algorithm upon greedy thought is given to discover communities in weighted network, thereby realizing the modularization of mechanical CAD assembly model. Finally, two CAD assembly models are employed to verify feasibility and effectiveness of the proposed method.
\end{abstract}

Keywords : CAD assembly model, Weighted network, Multi-source correlation information, Module partition, Community detection

\section{Introduction}

Modular design has been applied widely in complex product and mass customization design field (Bonvoisin et al., 2016). In product design phase, through analyzing the correlation relationships between parts or components from the aspects of function, structure and so on, the product can be divided into a series of modules with independent functions and stable structures to meet the various market requirements. It not only can greatly enhance product design efficiency and shorten product development cycle, but also reduce production costs and product management costs $(\mathrm{Gu}$ and Sosale, 1999). Meanwhile, With Computer-aided design (CAD) Systems used widely in industrial field, numerous three-dimensional (3D) CAD assembly models have been accumulated in enterprises, which embody plenty of design intents, function semantics and reusable modular structures knowledge. To provide support for modular design reuse and improve the reuse level of CAD assembly model, how to discover and identify the reusable modular structures in mechanical assembly model has become a vital issue in CAD assembly model reuse field.

In the product module partitioning process, the function and structure information are usually considered as the main factors to evaluating the correlation strength between components (Ulrich, 1994). To meet the different requirements of product module design, there have been some works dedicated to the product module partition. These works can be grouped into two main categories: graph-based module partition and matrix-based module partition. To solve the problem of product function modularization in product concept design phase, Kreng et al.(2004) analyzed the physical and functional relations among components, which was formatted by a component-to-component correlation matrix. The objective function of module partition was expressed as non-linear programming, and a grouping genetic algorithm was employed to search for the optimal module. Based on that, Gao et al.(2010) put forward a functional module identification method using generalized directed graphs and heuristics algorithm, where the three quantifiable rules (sequential flow rule, parallel flow rule and flow transformation rule) were defined, and a heuristics algorithm was formalized into rules to identify functional modules in function structure model. In order to meet concurrent design and enhance 3R-abilities (recycling, reuse and reduction), Huang et al.(2012) gave an approach for identifying the ideal 
product disassembly pattern and modules, where a component matrix was constructed to represent the relationships between product components by considering the disassembly pattern and 3R-abilities, and triangularization algorithm was applied to modularize a product. Helmer et al.(2010) described the interactions between components by using design structure matrices (DSMs), a DSM-based clustering algorithm was introduced for product clustering. Li et al.(2012) presented a three-phase clustering method for forming product modules to satisfy the demand of product collaborative design, in which function-parameter(FP) matrix was used to define dependency relationships between functions and parameters including shared parameter and protected parameter, then the three-phase clustering was given to find and estimate modular structure of the FP matrix for analyzing the information leakage from shared parameters to protected parameters.

3D assembly model implicating plenty of design intent, functional structures, key assembly structures(Han et al., 2017) and common design structures(Ma et al., 2010; Zhang et al., 2013) is important resource of model reuse in the product design process. Currently, there have been a few related works dedicated to assembly model retrieval(Chen et al., 2012; Deshmukh et al., 2008; Hu et al., 2013; Wang et al., 2016). But these methods work inaccurately and inefficiently on searching the CAD assembly models consisting of numerous parts in terms of complex assembly constraints and relationships, reuse level of which is not high. Based on modular idea, the CAD assembly model can be divided into several modular structures with function and stability, and then the similarity of the corresponding modules can be calculated concurrently. It can reduce effectively the complexity of assembly model retrieval and improve the assembly model reuse level. However, there are few research works on partitioning module in CAD assembly model. $\mathrm{Li}$ et al.(2015) proposed a hierarchical clustering for 3D CAD assembly model based on component dependencies to facilitate modular design of One-of-a-Kind Production (OKP). Firstly, assembly information was extracted and dependency strength between components was established by the relative DoF of components. Then the dependency relations between components was represented as DSM and a hierarchical clustering algorithm was used to identify modules in assembly model. But this method cannot satisfy well the realistic demand in product module design without considering function correlation between components and the hierarchical clustering algorithm is rather complex in its implementation.

To provide support for module retrieval concurrently, enhance retrieval efficiency and reuse level of assembly model, the paper aims to propose a method for partitioning automatically module of mechanical CAD assembly model, where correlation strength is evaluated from the point of view of multi-source correlation information, and then the correlation relatioships are expressed formally as a weighted network. Based on this, community detection is employed to find and evaluate the modular structure in mechanical assembly model.

The rest of this paper is organized as follows: the Systematic overview of the proposed approach is given in "Overview of approach" Section. In "Correlation analysis between assembled parts based on multi-source correlation information" Section, the correlation strength between assembled parts is evaluated based on multi-source correlation information. Subsequently, the detailed modeling process of weighted network for the correlation relationships between assembly parts is provided. In "Module partition for mechanical CAD assembly model" Section, the improved FN algorithm of community detection is put forward to partitioning module for assembly model. Following this Section, two cases are employed to analyze and verify feasibility of the proposed method in case study section. Finally, we conclude the paper and present further work directions.

\section{Overview of approach}

Fig. 1 shows the systematic overview of module partition for mechanical CAD assembly model. In the process of correlation relationships analysis between assembly parts, the multi-source correlation information (assembly structure, function, flow, material, etc.) is taken into account to evaluate respectively the correlation strength between parts. In the light of designers' special requirements, the comprehensive correlation strength can be calculated flexibly. After that, weighted network is employed to described explicitly and formally the correlation relationships between assembled parts in mechanical assembly model. Based on this, a community detection algorithm is given to find and evaluate the community structures in the weighed network, thereby the corresponding CAD assembly model partitioned into multiple modular structures.

The approach can evaluate completely and flexibly the correlation strength between parts, discover easily and conveniently higher quality and more realistic assembly modules in assembly model. It can not only decrease model modification when reusing assembly model, but also assist designers search more easily reusable modular structure to meet current reuse environment. 


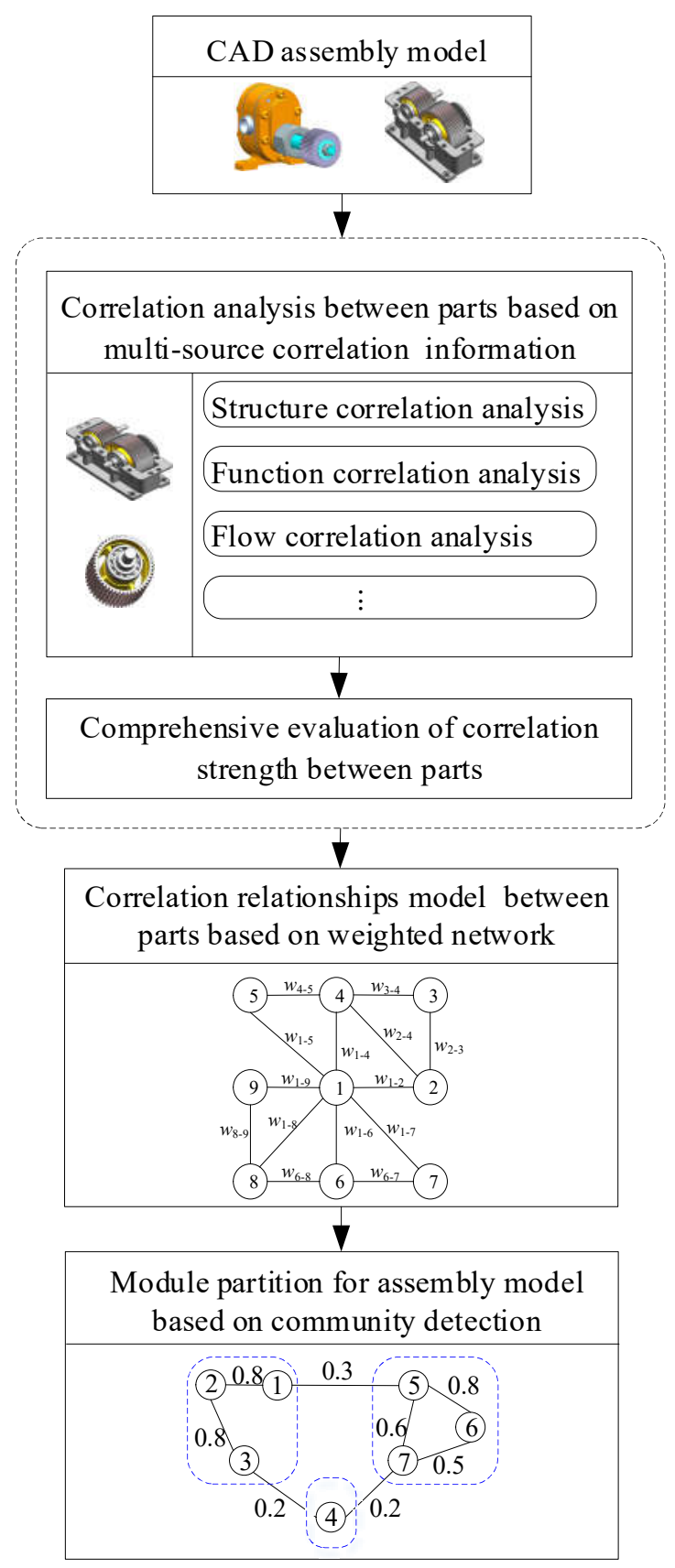

Fig. 1 Systematic overview of approach

\section{Correlation analysis between assembled parts based on multi-source correlation information}

Assembly is composed of a group of parts according to assembly relationships and assembly constraints information between adjacent parts, where each part has its own topology spatial position. Thus, the function and performance of assembly can be achieved. A mechanical CAD assembly model consists of several independent modular structures usually. The assembled parts that belong to the same module are equipped with higher correlation strength and can perform certain functions together.

Therefore, it is necessary to analyze the correlation strength between assembled parts in the process of module partition for mechanical CAD assembly model. The correlation strength between assembled parts is evaluated synthetically from the point of view of multi-source correlation information. Because the assembly structure, function and flows (material flow, energy flow and signal flow) have a vital influence on correlation strength between assembled parts, the correlation strength will be analyzed in detail from the aspects of above three correlation factors in the following. 


\subsection{Structure correlation analysis between assembled parts}

Table 1 Structure correlation strength evaluation between assembled parts

\begin{tabular}{|c|c|c|c|}
\hline No. & Connection type & Description & $I_{s}(i, j)$ \\
\hline 1 & $\begin{array}{l}\text { Connection where the parts } \\
\text { cannot be disassembled }\end{array}$ & $\begin{array}{l}\text { Rivet joint, welding, } \\
\text { bonding, etc. }\end{array}$ & 1 \\
\hline 2 & $\begin{array}{l}\text { Connection where the parts be } \\
\text { disassembled difficultly }\end{array}$ & $\begin{array}{l}\text { Interference fit, } \\
\text { threaded connection, etc. }\end{array}$ & 0.8 \\
\hline 3 & $\begin{array}{l}\text { Connection where the parts be } \\
\text { disassembled easily }\end{array}$ & $\begin{array}{l}\text { Pin connection, } \\
\text { key connection, etc. }\end{array}$ & 0.5 \\
\hline 4 & $\begin{array}{l}\text { Connection where the parts be } \\
\text { disassembled very easily }\end{array}$ & $\begin{array}{l}\text { clearance fit, surface } \\
\text { contact, etc. }\end{array}$ & 0.3 \\
\hline
\end{tabular}

The structure correlation strength between assembled parts is determined mainly by connection types or mating types between them. The structure correlation strength between assembled parts can be evaluated in terms of the disassembly and stability of connection types. Table 1 shows the structure correlation strength evaluation between assembled parts, where $I_{S}(i, j)$ represents structure correlation strength between part $i$ and part $j$.

\subsection{Function correlation analysis between assembled parts}

The product functions can be achieved by one or more parts together in CAD assembly model. Because one module is equipped with one function or several sub-functions, function correlation plays great influence on module partition. In addition, the parts between which function correlation strength is higher should be grouped into one module. The function correlation strength reflects mainly synergy degree of two or more parts achieving the same functions in assembly model. For example, shaft and key are combined together to achieve the function 'transfer power', and both of them indispensable. Then, the function correlation strength between shaft and key is strongest. Table 2 shows the function correlation strength evaluation between assembled parts, where $I_{F}(i, j)$ represents function correlation strength between part $i$ and part $j$.

Table 2 Function correlation strength evaluation between assembled parts

\begin{tabular}{ccccc}
\hline \hline No. & Correlation type & \multicolumn{1}{c}{ Description } & $I_{F}(i, j)$ \\
\hline 1 & Strongest & $\begin{array}{l}\text { The part } i \text { and part } j \text { achieve collectively and interdependently one } \\
\text { or more functions, both of them important. }\end{array}$ & 1 \\
& Stronger & $\begin{array}{l}\text { The part } i \text { and part } j \text { working collectively achieve one or more } \\
\text { functions, there is closer cooperative relationship between them. }\end{array}$ & 0.7 \\
3 & Strong & $\begin{array}{l}\text { The part } i \text { and part } j \text { working collectively achieve one or more } \\
\text { functions, there is close cooperative relationship between them. }\end{array}$ & 0.5 \\
& The part $i$ and part $j$ working collectively achieve one or more & 0.3 \\
4 & Weak & functions, there is weak cooperative relationship between them. & 0.3 \\
\hline
\end{tabular}

\subsection{Flow correlation analysis between assembled parts}

Flow correlation is analyzed by judging whether there exsists energy flow, material flow or signal flow between parts in assembly model. The flow correlation strength between assembled parts is also evaluated according to energy flow, material flow and signal flow. For example, there is 'torque transmission' betweem mating gears, it is considered that the flow correlation strength between them is strongest. Table 3 shows the flow correlation strength evaluation between assembled parts, where $I_{A}(i, j)$ represents flow correlation strength between part $i$ and part $j$.

Thing to note here is that the structure-function correlation analysis and functional semantics have been accomplished in CAD assembly model. Through structure-function correlation analysis, the function relationships and flow relationships between assembled parts have been obtained. Furthermore, the structure-function relation model is constructed based on polychromatic sets for expressing explicitly and formally relationships between functional 
structures, assembled parts and functional semantics. On the basis of that, the function and flow correlation strength between assembled parts can be analyzed and evaluated.

Table 3 Flow correlation strength evaluation between assembled parts

\begin{tabular}{clc}
\hline \hline No. & \multicolumn{1}{c}{ Description } & $I_{A}(i, j)$ \\
\hline 1 & $\begin{array}{l}\text { There exists energy flow between part } i \text { and part } j \text {, such as } \\
\text { torque transmission,etc. }\end{array}$ & 1 \\
2 & $\begin{array}{l}\text { There exists signal flow between part } i \text { and part } j \text {, such as } \\
\text { electricity transmission,etc. }\end{array}$ & 0.8 \\
3 & $\begin{array}{l}\text { There exists material flow between part } i \text { and part } j \text {, such as } \\
\text { solid, liquid, gas transmission,etc. }\end{array}$ & 0.4 \\
4 & There exists no flow between part $i$ and part $j$. & 0 \\
\hline
\end{tabular}

\subsection{Comprehensive evaluation of correlation strength between assembled parts}

Through the above correlation analysis between parts from the aspects of structure correlation, function correlation and flow correlation, the corresponding correlation strength can be obtained. Here, we give the weigh $\omega_{i}(i=1,2,3)$ that can reflect degree of structure correlation, function correlation and flow correlation influence on module partition. Moreover, the range of each weight is $[0,1]$ and their sum equals 1 , i.e. $\omega_{1}+\omega_{2}+\omega_{3}=1$. Due to difference of the structural characteristics of different mechanical products and design requirements, the weight values of structure correlation strength, function correlation strength and flow correlation strength are various when module partitioning of different categories of assembly models. There are two main factors to be considered for establishing weight values. They are as follows: product structure characteristics and design requirement.

(1) Product structure characteristics main embody the structure-function correlation relationships. There is nonlinear and complex relationship among structure correlation strength, function correlation strength and flow correlation strength in different categories of mechanical products. The structure characteristics are closer among similar products, and there are great differences among different categories of products. In general, the weight values of the three factors should be given by domain experts or experience knowledge in the process of module partition of different categories of mechanical assembly models.

(2) Design requirement mainly reflects designer/engineer's demand degree in structure, function and flow of assembled parts within modules. The factor is equipped with higher demand degree, the greater is the corresponding weight value.

Accordingly, the comprehensive correlation strength $I(i, j)$ can be expressed as follows:

$$
I(i, j)=I_{S}(i, j) \times \omega_{1}+I_{F}(i, j) \times \omega_{2}+I_{A}(i, j) \times \omega_{3}
$$

Where, $I_{s}(i, j), I_{F}(i, j)$ and $I_{A}(i, j)$ represent respectively the structure correlation strength, function correlation strength and flow correlation strength between assembled parts $i$ and $j . \omega_{1}, \omega_{2}$ and $\omega_{3}$ represent respectively weight of $I_{S}(i, j), \quad I_{F}(i, j)$ and $I_{A}(i, j)$, which are assigned in light of the specific circumstances. Based on comprehensive evaluation result, the correlation matrix $\mathbf{I}$ between assembled parts is a square matrix of order $n$, where $n$ is the number of parts assembled, which is given by

$$
\mathbf{I}=\left[\begin{array}{cccc}
1 & I(1,2) & \mathrm{L} & I(1, n) \\
I(1,2) & 1 & \mathrm{~L} & I(2, n) \\
\mathrm{M} & \mathrm{M} & 1 & \mathrm{M} \\
I(1, n) & I(2, n) & \mathrm{L} & 1
\end{array}\right]
$$

\section{Weighted network for correlation relationships between assembled parts}

Through correlation analysis between assembled parts based on multi-source correlation information, the 
comprehensive correlation strength matrix I can be evaluated and obtained. A weighted network can be employed to describe the correlation relationships between parts in mechanical CAD assembly model. It is constructed by superimposing the assembled parts, where the node denotes assembled part, the undirected edge and its weight denote respectively correlation relationship and correlation strength between assembled parts. Thus, the weighted network model is express by

$$
G=\{V, E, W\}
$$

Where, $V$ is a set of nodes which contains assembled parts, $E$ is a set of edges, each of which represents correlation relationship between parts, and $W$ a set of edges weight, each of which represents correlation strength of the corresponding edge in network $G$.

An example of a mechanical assembly is shown in Fig. 2. Fig. 2(a) shows cross-section of the assembly. The weighted network model for correlation relationships between parts is shown in Fig. 2(b).
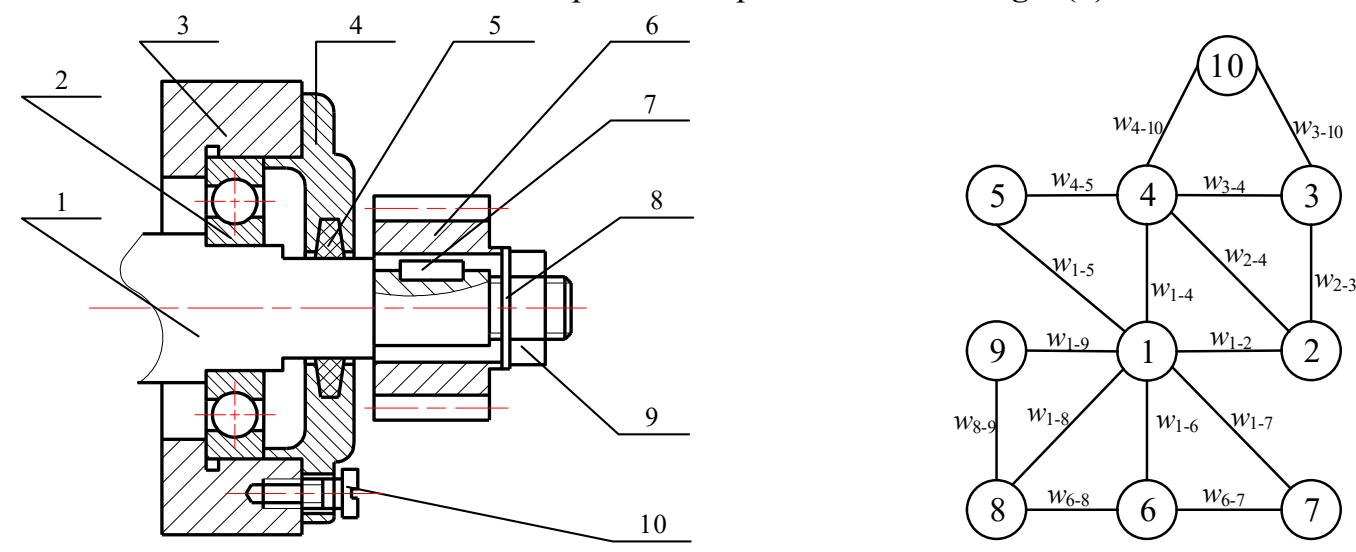

$\begin{array}{lll}\text { (a) Cross-section of mechanical CAD assembly model } & \text { (b) Weighted network for correlation relations between parts }\end{array}$

Fig. 2 An assembly model and its weighted network

\section{(1) Adjacency matrix A}

A weighted network $G$ can be represented mathematically by an $n \times n$ adjacency matrix $\mathbf{A}$ with elements

$$
A_{i j}=\left\{\begin{array}{cc}
\omega_{i j} & \text { if there is correlation relationship between parts } i \text { and } j . \\
0 & \text { otherwise }
\end{array}\right.
$$

Where, $\omega_{i j}$ represents weight of the edge $e_{i j}$, that is the comprehensive correlation strength $I_{i j}$ between part $i$ and $\operatorname{part} j$.

\section{(2) Modularity $Q$}

Modularity is a measure of the quality of division for a network. The modularity in an unweighted network is defined in Girvan Newman algorithm(Newman, 2004), which can be employed and extended to measure of the quality for community divisions in weighed network. The modularity $Q$ in weighed network can be given by

$$
\begin{gathered}
Q=\frac{1}{2 \omega} \sum_{i j}\left[\omega_{i j}-\frac{k_{i} k_{j}}{2 \omega}\right] \delta\left(C_{i}, C_{j}\right) \\
\omega=\frac{1}{2} \sum_{i j} \omega_{i j} \\
k_{i}=\sum_{j} \omega_{i j} \\
\Delta Q=\frac{1}{\omega}\left(\omega_{i j}-\frac{k_{i} k_{j}}{\omega}\right) \delta\left(C_{i}, C_{j}\right)
\end{gathered}
$$

Where, $\omega$ indicates the total sum of edges weight in the network $G, k_{i}$ indicates the sum of weight of edges connecting with node $i, \omega_{i j}$ indicates the sum of weight of edges connecting with nodes $i$ and $j, C_{i}$ represents the 
community to which node $i$ is assigned. The $\delta$ function $\delta\left(C_{i}, C_{j}\right)$ is 1 if $C_{i}=C_{j}$ and 0 otherwise. $\Delta Q$ represents the change in $Q$ upon joining two communities.

\section{Module partition for mechanical CAD assembly model}

The purpose of module partition is that the system is divided into several modules, which have dense internal links but between which there is only a lower density of external links. For a given assembly model, it can be partitioned flexibly into some modules with independent functions and stable structures in terms of special requirements of engineering designers and characteristics of product domain. Thus, the modular assembly structure and part models can be obtained to provide support for assembly model retrieval and reuse.

Community can characterize cluster effect existing in complex network, groups of nodes within which the connections are dense but between which they are sparser(Newman and Girvan, 2004). Community detection aims at finding and evaluating the community structure in network by analyzing the connections or similarity between nodes, which has been widely used in many fields, such as subassembly identification for truss structures(Cao et al., 2015), image segmentation(Mourchid et al., 2016), knowledge recommendation(Wang et al., 2016), etc. Community detection can be employed to identify the community structures in weighted network for correlation relations between assembled parts, where one community structure corresponds to a modular structure in mechanical CAD assembly model.

\subsection{Module partition based on Fast Newman}

Fast Newman (FN)(Newman, 2004) is a fasting algorithm for detecting community structure in network that can be used to discover the communities in correlation relationships network for assembled parts, thereby identifying and find modular structures in mechanical CAD assembly model.

In essence, FN algorithm falls in the category of agglomerative hierarchical clustering based on greedy idea, where each node is considered as the sole member of one of $n$ communities in network, and then repeatedly join communities together in pairs, choosing at each step the join that results in the greatest change $\Delta Q$ in modularity $Q$. Until the $n$ nodes form a community structure, i.e., original network G. The process of improved FN is given in detail for module partition of assembly model as follows.

FN Algorithm

Input: G: weighted network for correlation relationships between assembled parts,

A: the matrix corresponding comprehensive correlation strength between assembled parts

Output: $C$ : Community set, $Q$ : modularity function, $\mathrm{G}$ ': community structure

Step1: each node is considered as the sole member of one of $n$ communities in weighed network G, let the modularity function is $Q$.

Step2: the total sum of edges weight $\omega$ in G, the sum of edges weight of node $i$ and the sum of weight of edges connecting nodes $i, j$ in each community are calculated.

Step3: join communities together in pairs, the change $\Delta Q$ in modularity $Q$ upon joining two communities is calculated.

Step4: the join that results in the greatest increase $\Delta Q$ is chosen, which is considered as a new community structure. Let number of communities $n=n-1$.

Step5: the new communities form a new network $\mathrm{G}$, let $\mathrm{Q}=\mathrm{Q}+\Delta Q$.

Step6: judge the number of communities $n$, if $n>1$, go to step2-step5. Otherwise, then end.

\subsection{Quality evaluation of module partition}

The FN algorithm for module partition can produce some division of the weighted network into communities, where the output is in the form of a dendrogram representing an entire nested hierarchy of possible community divisions for the weighted network G. It is very necessary for us to know when the communities (modules) found by the FN algorithm are good ones. The modularity $Q$ is employed as a measure of the quality of a division of weighted network $\mathrm{G}$. The value of $Q$ for each split of weighted network $\mathrm{G}$ into communities is higher, the corresponding communities are better. In practice, the max value of $Q$ falls in the range of 0.3 to 0.7 . There are one or two local peaks in the value of $Q$, the positions of which indicate satisfactory splits for the weighted network G. In the meanwhile, the dendrogram is cut to get a sensible division of the network $G$, where the communities split correspond respectively to the expected modules for mechanical CAD assembly model. 


\section{Case study}

\subsection{Case I}

In this Section, CAD assembly model of a gear oil pump is presented to analyze the correlation relationships between assembled parts, identify modular structure with some function and reusability. Fig. 3 shows Cross-section of the gear oil pump assembly model, parts list of the assembly is shown in Table $\mathbf{4}$ and Table $\mathbf{5}$ shows the connection information between assembled parts extracted from CAD assembly model.

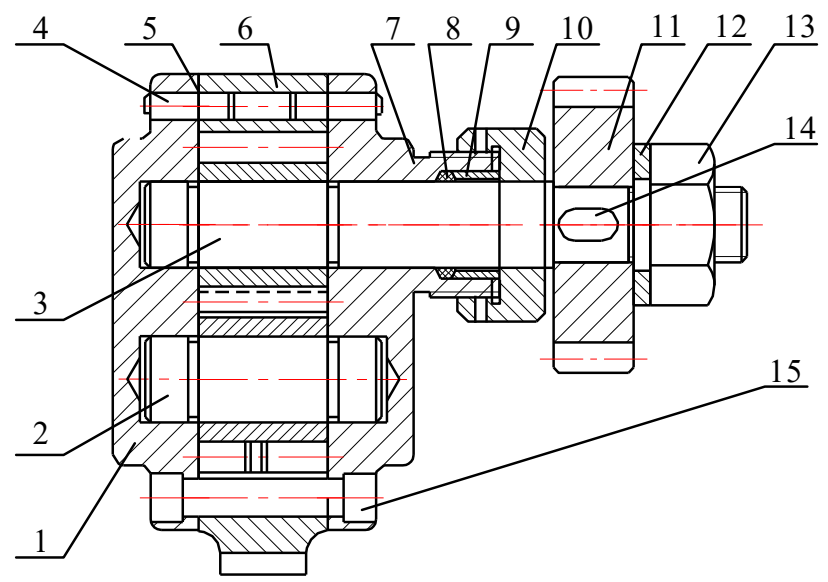

Fig. 3 Cross-section of the gear oil pump assembly model

Table 4 Parts list of the gear oil pump assembly model

\begin{tabular}{cccc|cccc}
\hline \hline No. & Name & Quantity & Material & No. & Name & Quantity & Material \\
\hline 1 & Left pump cover & 1 & HT200 & 9 & Bush & 1 & C45 \\
2 & Driven gear shaft & 1 & C45 & 10 & Compression nut & 1 & Q235-A \\
3 & Driving gear shaft & 1 & C45 & 11 & Driving gear & 1 & C45 \\
4 & Cylindrical pin & 4 & Q235-A & 12 & Washer & 1 & Q235-A \\
5 & Gasket & 2 & Paper & 13 & Nut & 1 & Q235-A \\
6 & Pump body & 1 & HT200 & 14 & Key & 1 & C45 \\
7 & Right pump cover & 1 & HT200 & 15 & Screw & 12 & Q235-A \\
8 & Seal & 1 & Rubber & & & & \\
\hline
\end{tabular}

Table 5 The connection information between assembled parts

\begin{tabular}{cl|cl|cl}
\hline \hline Parts pair & Connection type & Parts pair & Connection type & Parts pair & Connection type \\
\hline $1-2$ & Clearance fit & $3-11$ & Surface contact & $7-8$ & Surface contact \\
$1-3$ & Clearance fit & $3-12$ & Clearance fit & $7-9$ & Surface contact \\
$1-4$ & Pin connection & $3-13$ & Threaded connection & $7-10$ & Threaded connection \\
$1-5$ & Surface contact & $3-14$ & Key connection & $7-15$ & Threaded connection \\
$1-6$ & Alignment & $4-5$ & Pin connection & $8-9$ & Surface contact \\
$1-15$ & Threaded connection & $4-6$ & Pin connection & $9-10$ & Surface contact \\
$2-3$ & mating gears & $4-7$ & Pin connection & $11-12$ & Surface contact \\
$2-7$ & Clearance fit & $5-6$ & Surface contact & $11-14$ & Key connection \\
$3-7$ & Clearance fit & $5-7$ & Surface contact & $12-13$ & Surface contact \\
$3-8$ & Surface contact & $6-7$ & Alignment & & \\
$3-9$ & Surface contact & $6-15$ & Threaded connection & & \\
\hline
\end{tabular}

\subsubsection{Correlation analysis between assembled parts}

It is a vital step to analyze the correlation relationships between parts when evaluating the correlation strength 
based on multi-source correlation information. Here, we assume that the connection type and functional semantics information have been obtained from CAD assembly model ahead of time(Mathew and Rao, 2010). Then the Tables 1-3 mentioned are used to evaluate the correlation strength between parts. The evaluation result of correlation strength is shown in Fig. 4, where Fig. 4(a) shows the structure correlation strength matrix $I_{S}$, Fig. 4(b) shows function correlation strength matrix $\mathrm{I}_{F}$ and Fig. 4(c) shows flow correlation strength matrix $\mathrm{I}_{A}$.

\begin{tabular}{|c|c|c|c|c|c|c|c|c|c|c|c|c|c|c|c|}
\hline & $\mathbf{1}$ & $\mathbf{2}$ & $\mathbf{3}$ & $\mathbf{4}$ & $\mathbf{5}$ & $\mathbf{6}$ & $\mathbf{7}$ & $\mathbf{8}$ & $\mathbf{9}$ & $\mathbf{1 0}$ & $\mathbf{1 1}$ & $\mathbf{1 2}$ & $\mathbf{1 3}$ & $\mathbf{1 4}$ & $\mathbf{1 5}$ \\
\hline $\mathbf{1}$ & 0 & 0.3 & 0.3 & 0.5 & 0.3 & 0.3 & 0 & 0 & 0 & 0 & 0 & 0 & 0 & 0 & 0.8 \\
\hline $\mathbf{2}$ & 0.3 & 0 & 0.3 & 0 & 0 & 0 & 0.3 & 0 & 0 & 0 & 0 & 0 & 0 & 0 & 0 \\
\hline $\mathbf{3}$ & 0.3 & 0.3 & 0 & 0 & 0 & 0 & 0.3 & 0.3 & 0.3 & 0 & 0.3 & 0.8 & 0.8 & 0.5 & 0 \\
\hline $\mathbf{4}$ & 0.5 & 0 & 0 & 0 & 0.5 & 0.5 & 0.5 & 0 & 0 & 0 & 0 & 0 & 0 & 0 & 0.8 \\
\hline $\mathbf{5}$ & 0.3 & 0 & 0 & 0.5 & 0 & 0.3 & 0.3 & 0 & 0 & 0 & 0 & 0 & 0 & 0 & 0 \\
\hline $\mathbf{6}$ & 0.3 & 0 & 0 & 0.5 & 0.3 & 0 & 0.3 & 0 & 0 & 0 & 0 & 0 & 0 & 0 & 0.8 \\
\hline $\mathbf{7}$ & 0 & 0.3 & 0.3 & 0.5 & 0.3 & 0.3 & 0 & 0.3 & 0.3 & 0.8 & 0 & 0 & 0 & 0 & 0.8 \\
\hline $\mathbf{8}$ & 0 & 0 & 0.3 & 0 & 0 & 0 & 0.3 & 0 & 0.3 & 0 & 0 & 0 & 0 & 0 & 0 \\
\hline $\mathbf{9}$ & 0 & 0 & 0.3 & 0 & 0 & 0 & 0.3 & 0.3 & 0 & 0.3 & 0 & 0 & 0 & 0 & 0 \\
\hline $\mathbf{1 0}$ & 0 & 0 & 0.8 & 0 & 0 & 0 & 0.8 & 0 & 0.3 & 0 & 0 & 0 & 0 & 0 & 0 \\
\hline $\mathbf{1 1}$ & 0 & 0 & 0.3 & 0 & 0 & 0 & 0 & 0 & 0 & 0 & 0 & 0.3 & 0 & 0.5 & 0 \\
\hline $\mathbf{1 2}$ & 0 & 0 & 0.8 & 0 & 0 & 0 & 0 & 0 & 0 & 0 & 0.3 & 0 & 0.3 & 0 & 0 \\
\hline $\mathbf{1 3}$ & 0 & 0 & 0.8 & 0 & 0 & 0 & 0 & 0 & 0 & 0 & 0 & 0.3 & 0 & 0 & 0 \\
\hline $\mathbf{1 4}$ & 0 & 0 & 0.5 & 0 & 0 & 0 & 0 & 0 & 0 & 0 & 0.5 & 0 & 0 & 0 & 0 \\
\hline $\mathbf{1 5}$ & 0.8 & 0 & 0 & 0.5 & 0 & 0.8 & 0.8 & 0 & 0 & 0 & 0 & 0 & 0 & 0 & 0 \\
\hline
\end{tabular}

(a) Structure correlation strength matrix $\mathrm{I}_{S}$

\begin{tabular}{|c|c|c|c|c|c|c|c|c|c|c|c|c|c|c|c|}
\hline & $\mathbf{1}$ & $\mathbf{2}$ & $\mathbf{3}$ & $\mathbf{4}$ & $\mathbf{5}$ & $\mathbf{6}$ & $\mathbf{7}$ & $\mathbf{8}$ & $\mathbf{9}$ & $\mathbf{1 0}$ & $\mathbf{1 1}$ & $\mathbf{1 2}$ & $\mathbf{1 3}$ & $\mathbf{1 4}$ & $\mathbf{1 5}$ \\
\hline $\mathbf{1}$ & 0 & 0.5 & 0.5 & 0.7 & 0.3 & 1 & 1 & 0 & 0 & 0 & 0 & 0 & 0 & 0 & 0.7 \\
\hline $\mathbf{2}$ & 0.5 & 0 & 1 & 0 & 0 & 0.5 & 0.5 & 0 & 0 & 0 & 0 & 0 & 0 & 0 & 0 \\
\hline $\mathbf{3}$ & 0.5 & 1 & 0 & 0 & 0 & 0.5 & 0.5 & 1 & 0.7 & 0.5 & 1 & 0.5 & 1 & 1 & 0 \\
\hline $\mathbf{4}$ & 0.7 & 0 & 0 & 0 & 0.7 & 0.7 & 0.7 & 0 & 0 & 0 & 0 & 0 & 0 & 0 & 0 \\
\hline $\mathbf{5}$ & 0.3 & 0 & 0 & 0.7 & 0 & 0.3 & 0.3 & 0 & 0 & 0 & 0 & 0 & 0 & 0 & 0.7 \\
\hline $\mathbf{6}$ & 1 & 0.5 & 0.5 & 0.7 & 0.3 & 0 & 0.7 & 0 & 0 & 0 & 0 & 0 & 0 & 0 & 0.7 \\
\hline $\mathbf{7}$ & 1 & 0.5 & 0.5 & 0.7 & 0.3 & 0.7 & 0 & 1 & 0.7 & 1 & 0 & 0 & 0 & 0 & 0 \\
\hline $\mathbf{8}$ & 0.5 & 0 & 1 & 0 & 0 & 0 & 1 & 0 & 0.7 & 0.3 & 0 & 0 & 0 & 0 & 0 \\
\hline $\mathbf{9}$ & 0.7 & 0 & 0.7 & 0 & 0 & 0 & 0.7 & 0.7 & 0 & 0.7 & 0 & 0 & 0 & 0 & 0 \\
\hline $\mathbf{1 0}$ & 0.3 & 0 & 0.5 & 0 & 0 & 0 & 1 & 0.3 & 0.7 & 0 & 0 & 0 & 0 & 0 & 0 \\
\hline $\mathbf{1 1}$ & 0 & 0 & 1 & 0 & 0 & 0 & 0 & 0 & 0 & 0 & 0 & 0.7 & 0.7 & 1 & 0 \\
\hline $\mathbf{1 2}$ & 0.7 & 0 & 0.5 & 0 & 0 & 0 & 0 & 0 & 0 & 0 & 0.7 & 0 & 1 & 0 & 0 \\
\hline $\mathbf{1 3}$ & 0 & 0 & 1 & 0 & 0 & 0 & 0 & 0 & 0 & 0 & 0.7 & 1 & 0 & 0 & 0 \\
\hline $\mathbf{1 4}$ & 0 & 0 & 1 & 0 & 0 & 0 & 0 & 0 & 0 & 0 & 1 & 0 & 0 & 0 & 0 \\
\hline $\mathbf{1 5}$ & 0.7 & 0 & 0 & 0 & 0.7 & 0.7 & 0 & 0 & 0 & 0 & 0 & 0 & 0 & 0 & 0 \\
\hline
\end{tabular}

(b) Functions correlation strength matrix $\mathrm{I}_{F}$

\begin{tabular}{|c|c|c|c|c|c|c|c|c|c|c|c|c|c|c|c|}
\hline & $\mathbf{1}$ & $\mathbf{2}$ & $\mathbf{3}$ & $\mathbf{4}$ & $\mathbf{5}$ & $\mathbf{6}$ & $\mathbf{7}$ & $\mathbf{8}$ & $\mathbf{9}$ & $\mathbf{1 0}$ & $\mathbf{1 1}$ & $\mathbf{1 2}$ & $\mathbf{1 3}$ & $\mathbf{1 4}$ & $\mathbf{1 5}$ \\
\hline $\mathbf{1}$ & 0 & 0.4 & 0 & 1 & 0.4 & 0.4 & 0.4 & 0 & 0 & 0 & 0 & 0 & 0 & 0 & 1 \\
\hline $\mathbf{2}$ & 0.4 & 0 & 1 & 0 & 0 & 0.4 & 0.4 & 0 & 0 & 0 & 0 & 0 & 0 & 0 & 0 \\
\hline $\mathbf{3}$ & 0 & 1 & 0 & 0 & 0 & 0.4 & 0.4 & 0 & 0 & 0 & 1 & 0 & 1 & 1 & 0 \\
\hline $\mathbf{4}$ & 1 & 0 & 0 & 0 & 1 & 1 & 1 & 0 & 0 & 0 & 0 & 0 & 0 & 0 & 0 \\
\hline $\mathbf{5}$ & 0.4 & 0 & 0 & 1 & 0 & 0.4 & 0.4 & 0 & 0 & 0 & 0 & 0 & 0 & 0 & 1 \\
\hline $\mathbf{6}$ & 0.4 & 0.4 & 0.4 & 1 & 0.4 & 0 & 0.4 & 0.4 & 0 & 0 & 0 & 0 & 0 & 0 & 1 \\
\hline $\mathbf{7}$ & 0.4 & 0.4 & 0.4 & 1 & 0.4 & 0.4 & 0 & 0.4 & 0.4 & 1 & 0 & 0 & 0 & 0 & 1 \\
\hline $\mathbf{8}$ & 0 & 0 & 0 & 0 & 0 & 0.4 & 0.4 & 0 & 1 & 1 & 0 & 0 & 0 & 0 & 0 \\
\hline $\mathbf{9}$ & 0 & 0 & 0 & 0 & 0 & 0 & 0.4 & 1 & 0 & 1 & 0 & 0 & 0 & 0 & 0 \\
\hline $\mathbf{1 0}$ & 0 & 0 & 0 & 0 & 0 & 0 & 1 & 1 & 1 & 0 & 0 & 0 & 0 & 0 & 0 \\
\hline $\mathbf{1 1}$ & 0 & 0 & 1 & 0 & 0 & 0 & 0 & 0 & 0 & 0 & 0 & 1 & 0 & 1 & 0 \\
\hline $\mathbf{1 2}$ & 0 & 0 & 0 & 0 & 0 & 0 & 0 & 0 & 0 & 0 & 1 & 0 & 1 & 0 & 0 \\
\hline $\mathbf{1 3}$ & 0 & 0 & 1 & 0 & 0 & 0 & 0 & 0 & 0 & 0 & 0 & 1 & 0 & 0 & 0 \\
\hline $\mathbf{1 4}$ & 0 & 0 & 1 & 0 & 0 & 0 & 0 & 0 & 0 & 0 & 1 & 0 & 0 & 0 & 0 \\
\hline $\mathbf{1 5}$ & 1 & 0 & 0 & 0 & 1 & 1 & 1 & 0 & 0 & 0 & 0 & 0 & 0 & 0 & 0 \\
\hline
\end{tabular}

(c) Flow correlation strength matrix $\mathrm{I}_{A}$

Fig. 4 Correlation strength evaluation between parts based on multi-source correlation information

\begin{tabular}{|c|c|c|c|c|c|c|c|c|c|c|c|c|c|c|c|}
\hline & $\mathbf{1}$ & $\mathbf{2}$ & $\mathbf{3}$ & $\mathbf{4}$ & $\mathbf{5}$ & $\mathbf{6}$ & $\mathbf{7}$ & $\mathbf{8}$ & $\mathbf{9}$ & $\mathbf{1 0}$ & $\mathbf{1 1}$ & $\mathbf{1 2}$ & $\mathbf{1 3}$ & $\mathbf{1 4}$ & $\mathbf{1 5}$ \\
\hline $\mathbf{1}$ & 0 & 0.38 & 0.30 & 0.66 & 0.32 & 0.53 & 0.38 & 0 & 0 & 0 & 0 & 0 & 0 & 0 & 0.81 \\
\hline $\mathbf{2}$ & 0.38 & 0 & 0.65 & 0 & 0 & 0.23 & 0.38 & 0 & 0 & 0 & 0 & 0 & 0 & 0 & 0 \\
\hline $\mathbf{3}$ & 0.30 & 0.65 & 0 & 0 & 0 & 0.23 & 0.38 & 0.45 & 0.36 & 0.15 & 0.65 & 0.55 & 0.90 & 0.75 & 0 \\
\hline $\mathbf{4}$ & 0.66 & 0 & 0 & 0 & 0.66 & 0.66 & 0.66 & 0 & 0 & 0 & 0 & 0 & 0 & 0 & 0 \\
\hline $\mathbf{5}$ & 0.32 & 0 & 0 & 0.66 & 0 & 0.32 & 0.32 & 0 & 0 & 0 & 0 & 0 & 0 & 0 & 0.41 \\
\hline $\mathbf{6}$ & 0.53 & 0.23 & 0.23 & 0.66 & 0.32 & 0 & 0.44 & 0.08 & 0 & 0 & 0 & 0 & 0 & 0 & 0.81 \\
\hline $\mathbf{7}$ & 0.38 & 0.38 & 0.38 & 0.66 & 0.32 & 0.44 & 0 & 0.53 & 0.44 & 0.90 & 0 & 0 & 0 & 0 & 0.6 \\
\hline $\mathbf{8}$ & 0 & 0 & 0.45 & 0 & 0 & 0.08 & 0.53 & 0 & 0.56 & 0.29 & 0 & 0 & 0 & 0 & 0 \\
\hline $\mathbf{9}$ & 0 & 0 & 0.36 & 0 & 0 & 0 & 0.44 & 0.56 & 0 & 0.56 & 0 & 0 & 0 & 0 & 0 \\
\hline $\mathbf{1 0}$ & 0 & 0 & 0.55 & 0 & 0 & 0 & 0.9 & 0.29 & 0.56 & 0 & 0 & 0 & 0 & 0 & 0 \\
\hline $\mathbf{1 1}$ & 0 & 0 & 0.65 & 0 & 0 & 0 & 0 & 0 & 0 & 0 & 0 & 0.56 & 0.21 & 0.75 & 0 \\
\hline $\mathbf{1 2}$ & 0 & 0 & 0.55 & 0 & 0 & 0 & 0 & 0 & 0 & 0 & 0.56 & 0 & 0.65 & 0 & 0 \\
\hline $\mathbf{1 3}$ & 0 & 0 & 0.9 & 0 & 0 & 0 & 0 & 0 & 0 & 0 & 0.21 & 0.65 & 0 & 0 & 0 \\
\hline $\mathbf{1 4}$ & 0 & 0 & 0.75 & 0 & 0 & 0 & 0 & 0 & 0 & 0 & 0.75 & 0 & 0 & 0 & 0 \\
\hline $\mathbf{1 5}$ & 0.81 & 0 & 0 & 0 & 0.41 & 0.81 & 0.60 & 0 & 0 & 0 & 0 & 0 & 0 & 0 & 0 \\
\hline
\end{tabular}

Fig. 5 Comprehensive correlation strength matrix I

Meanwhile, the weight of each correlation factor could be established in terms of the experience knowledge and the gear oil assembly characteristics. Let $\omega_{1}=0.5, \omega_{2}=0.3, \omega_{3}=0.2$, the better evaluation result will be achieved. On the basis of the analysis and evaluation of structure correlation, function correlation and flow correlation between assembled parts, the comprehensive correlation strength is calculated by the formula (1). Fig. $\mathbf{5}$ shows the 
comprehensive correlation strength matrix I.

\subsubsection{Weighted network model}

Based on comprehensive correlation strength matrix I, the weighted network $G$ for correlation relationship between parts in gear oil pump assembly model is constructed as shown in Fig. 6, where the node $v_{i}$ represents the part $i$ and $w_{i j}$ represents the comprehensive correlation strength between part $i$ and $\operatorname{part} j$.

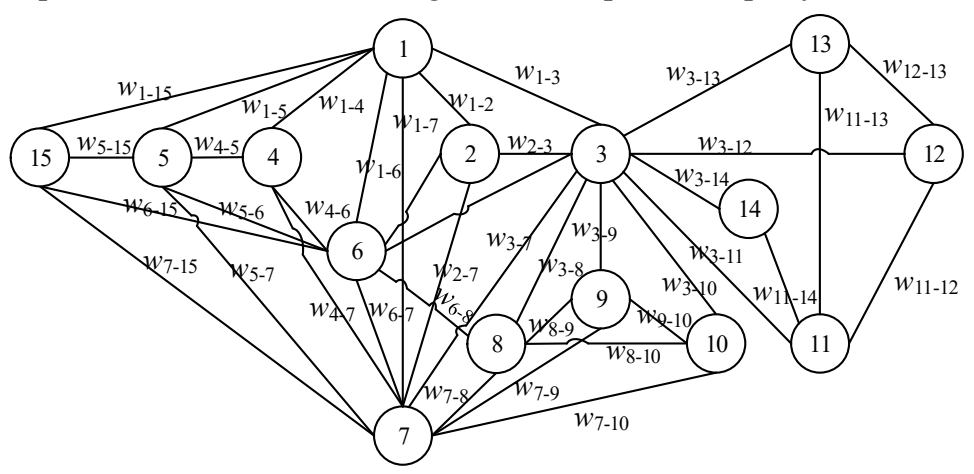

Fig. 6 Weighted network $\mathrm{G}$ for correlation relationship between parts

\subsubsection{Module partition of gear oil pump assembly model}

The FN algorithm given in Section 5 and Girvan Newman (GN)(Girvan and Newman, 2002) are used to community discovery for weighted network $\mathrm{G}$ in MATLAB. The module partition results and modularity $Q$ respectively corresponding the above two algorithms for the given gear oil pump assembly are shown in Fig. 7, where $k$ represents the number of communities or modules. Fig. 7(a) shows the dendrogram of module partition based on FN, Fig. 7(b) shows the dendrogram of module partition based on GN, and the modularity $Q$ for FN and GN is shown in Fig. 7(c). As shown in Fig. 7, the optimal modularity is $Q=0.3859$ and corresponds to the division into 3 communities for FN algorithm. And optimal modularity is $Q=0.3269$ and corresponds to the division into 4 communities for GN algorithm.

By contrast, the modularity value in FN is always higher than in GN as seen in Fig. 7(c), it means that the better module partition can be obtained by adopting FN algorithm. Therefore, the desired module partition of the gear oil pump assembly model is achieved when the number of modules is 3 , and the part nodes set $C_{1}=\left\{v_{2}, v_{3}, v_{11}, v_{12}, v_{13}\right.$, $\left.v_{14}\right\}, C_{2}=\left\{v_{1}, v_{4}, v_{5}, v_{6}, v_{15}\right\}$ and $C_{3}=\left\{v_{7}, v_{8}, v_{9}, v_{10}\right\}$ can be obtained corresponding to the driving gear shaft-gear module, pump body-left pump cover module and right pump cover-seal module, as shown Table 6. The modules partition result of gear oil pump assembly model is appropriate for realistic situation. For example, the module $C_{1}$ representing the driving gear shaft-gear module includes driving gear shaft, driving gear, driven gear shaft that are related to transmission functions and between which the correlation relationship is closer in the aspects of assembly, function and design parameter.

One thing to note is that the module partition result is also established in the light of the desired number of modules $k$ besides the modularity $Q$. The desired $k$ can be given by designers to meet their individual demand.
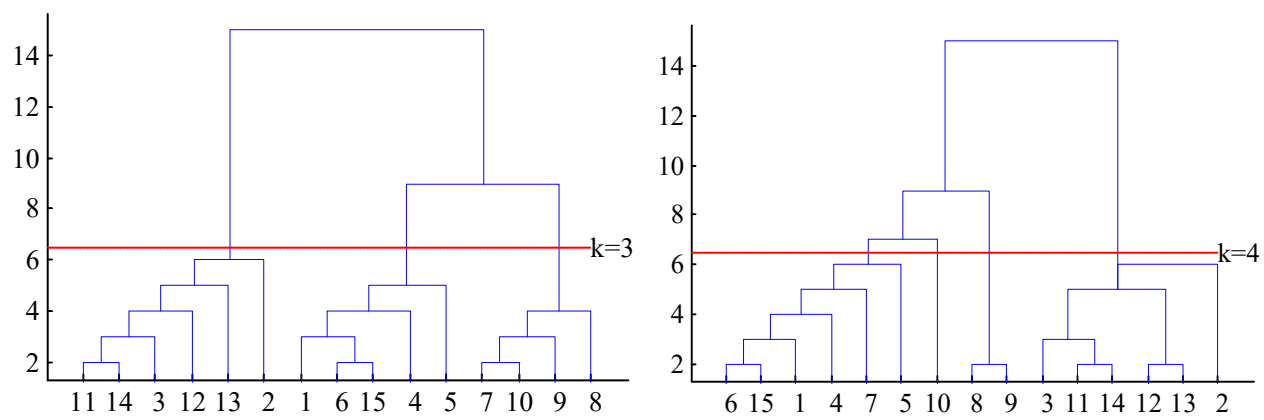

(a) The dendrogram of module partition based on FN

(b) The dendrogram of module partition based on GN 


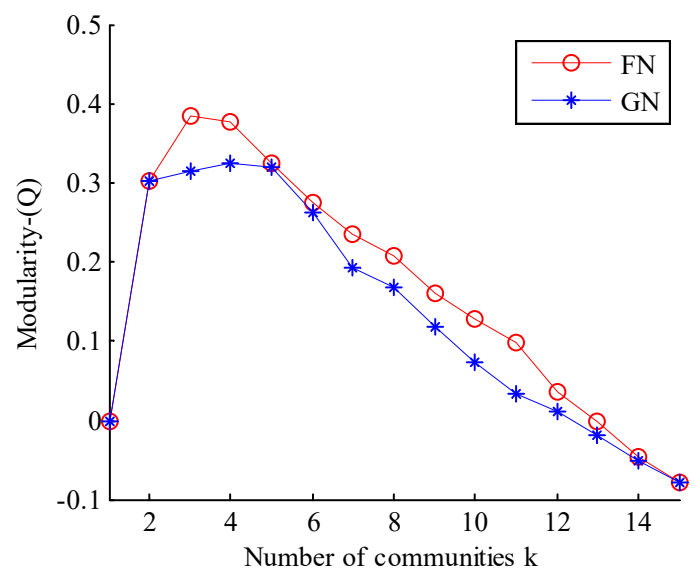

(c) The modularity $Q$ for FN and GN

Fig. 7 The module partition results and modularity $Q$ for gear oil pump assembly model

Table 6 Module partition result of the gear oil pump assembly model $(k=3)$

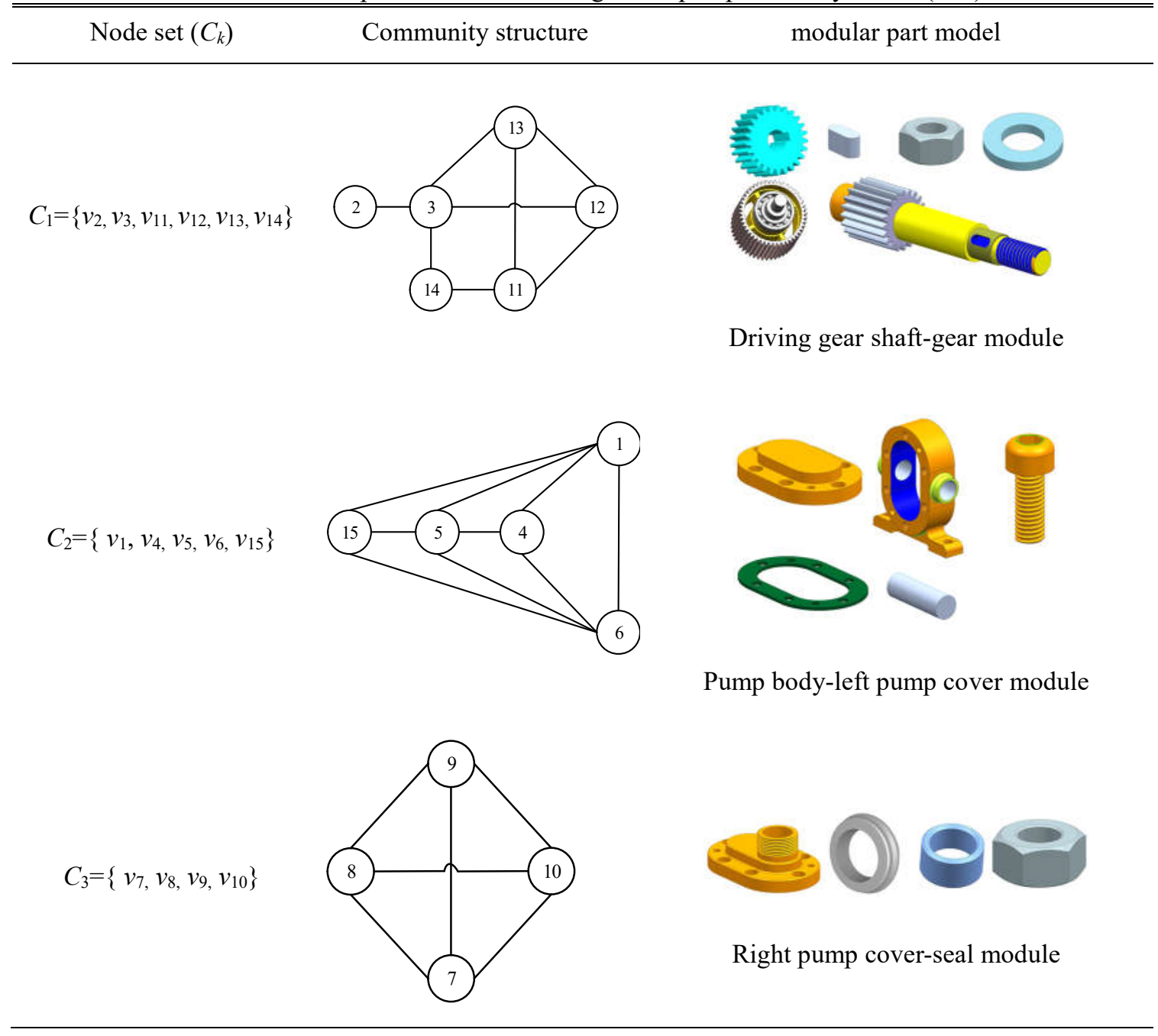

\subsection{Case II}

In the Section, an automobile differential assembly model is employed to testify effectiveness of the proposed method further. Fig. 8 shows Cross-section of the automobile differential assembly model, and parts list of the assembly is shown in Table 7. 

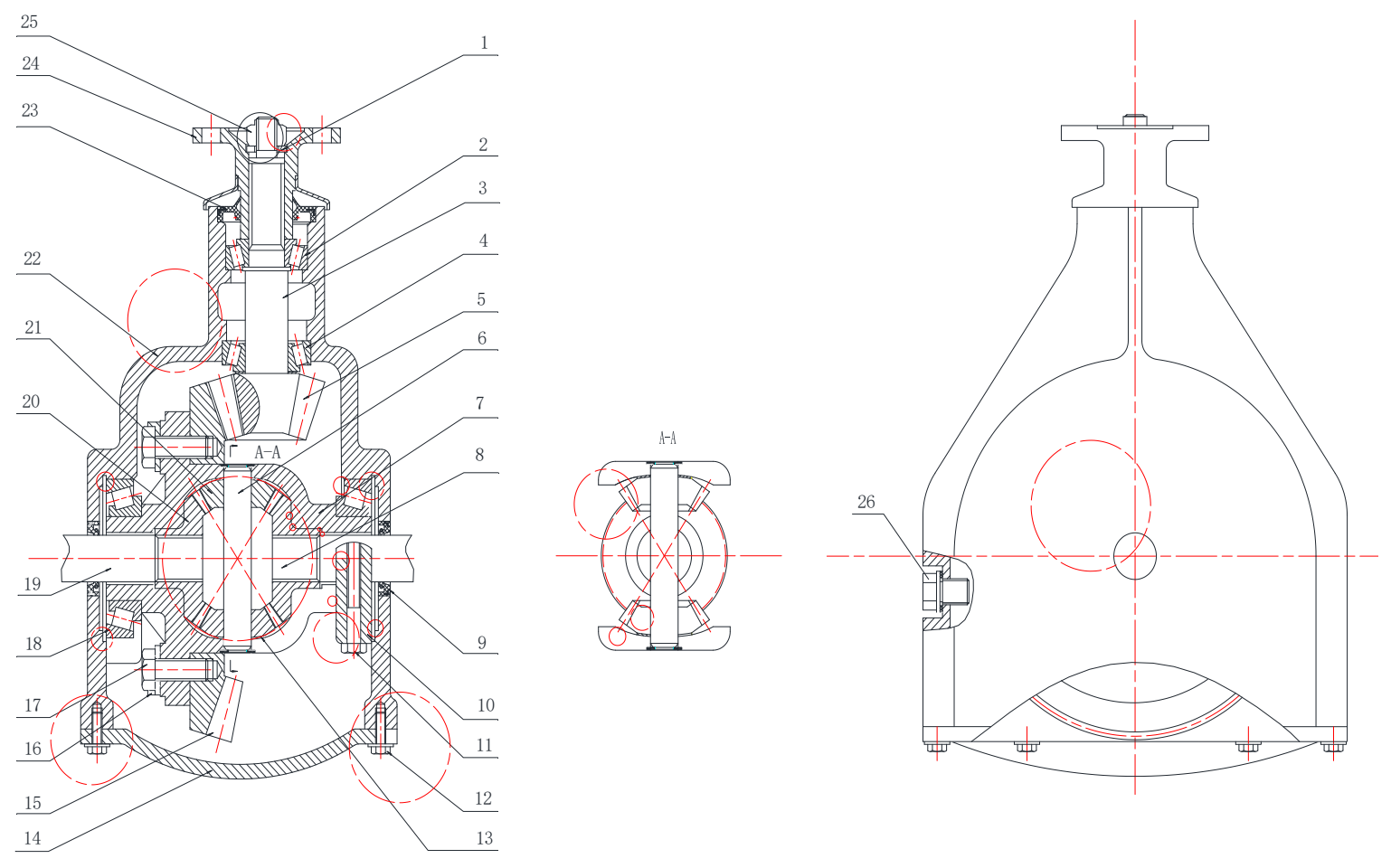

Fig. 8 Cross-section of the automobile differential assembly model

Table 7 Parts list of the automobile differential assembly model

\begin{tabular}{|c|c|c|c|c|c|c|c|}
\hline No. & Name & Quantity & Material & No. & Name & Quantity & Material \\
\hline 1 & Gaskets & 1 & $\mathrm{C} 45$ & 14 & Rear cover & 1 & $\mathrm{C} 45$ \\
\hline 2 & $\begin{array}{l}\text { Anterior tapered } \\
\text { roller bearing }\end{array}$ & 1 & $\mathrm{C} 35$ & 15 & Driven bevel gear & 1 & 20CrMnTi \\
\hline 3 & Driving shaft & 1 & $\mathrm{C} 45$ & 16 & Lock blade & 8 & 08 \\
\hline 4 & $\begin{array}{l}\text { Posterior tapered } \\
\text { roller bearing }\end{array}$ & 1 & $\mathrm{C} 35$ & 17 & Bolt & 8 & $40 \mathrm{Cr}$ \\
\hline 5 & Driving bevel gear & 1 & 20CrMnTi & 18 & Tapered roller bearing & 2 & C35 \\
\hline 6 & Planetary gear shaft & 1 & $40 \mathrm{Cr}$ & 19 & Output shaft & & $\mathrm{C} 45$ \\
\hline 7 & Differential carrier & 1 & QT450-10 & 20 & Semi-axle bevel gear & 2 & 20CrMnTi \\
\hline 8 & Output shaft & 1 & $\mathrm{C} 45$ & 21 & Planetary gear & 2 & 20CrMnTi \\
\hline 9 & Seal & 2 & Rubber & 22 & Outer shell & 1 & QT450-10 \\
\hline 10 & Bearing cover & 2 & QT450-10 & 23 & Seal & 1 & Rubber \\
\hline 11 & Bolt & 4 & $40 \mathrm{Cr}$ & 24 & Flange & 1 & $40 \mathrm{Cr}$ \\
\hline 12 & Bolt & 8 & $40 \mathrm{Cr}$ & 25 & Nut & 1 & $\mathrm{C} 45$ \\
\hline 13 & Spherical washer & 1 & 08 & 26 & Oil plug & 1 & SUS304 \\
\hline
\end{tabular}

\subsubsection{Correlation analysis between assembled parts}

In the same way as Case I, the Tables 1-3 previsouly mentioned are used to evaluate the correlation strength between parts. The weight of each correlation factor are established in terms of the experience knowledge and automobile differential assembly characteristics. Let $\omega_{1}=0.4, \omega_{2}=0.4, \omega_{3}=0.2$, the comprehensive correlation strength matrix I is obtained as shown in Fig. 9. 


\begin{tabular}{|c|c|c|c|c|c|c|c|c|c|c|c|c|c|c|c|c|c|c|c|c|c|c|c|c|c|c|}
\hline & 1 & 2 & 3 & 4 & 5 & 6 & 7 & 8 & 9 & 10 & 11 & 12 & 13 & 14 & 15 & 16 & 17 & 18 & 19 & 20 & 21 & 22 & 23 & 24 & 25 & 26 \\
\hline 1 & 0 & 0 & \begin{tabular}{|l|}
0.24 \\
\end{tabular} & 0 & 0 & 0 & 0 & 0 & 0 & 0 & 0 & 0 & 0 & 0 & 0 & 0 & 0 & 0 & 0 & 0 & 0 & 0 & 0 & \begin{tabular}{|l|l|}
0.24 \\
\end{tabular} & 0.88 & 0 \\
\hline 2 & 0 & 0 & \begin{tabular}{|l}
0.8 \\
\end{tabular} & 0 & 0 & 0 & 0 & 0 & 0 & 0 & 0 & 0 & 0 & 0 & 0 & 0 & 0 & 0 & 0 & 0 & 0 & \begin{tabular}{|l|}
0.8 \\
\end{tabular} & 0 & \begin{tabular}{|l|l|} 
\\
\end{tabular} & 0 & 0 \\
\hline 3 & 0.24 & 0.8 & 0 & 0.8 & 0.32 & 0 & 0 & 0 & 0 & 0 & 0 & 0 & 0 & 0 & 0 & 0 & 0 & 0 & 0 & 0 & 0 & 0 & 0 & 0 & 0.44 & 0 \\
\hline 4 & 0 & 0 & 0.8 & 0 & 0.12 & 0 & 0 & 0 & 0 & 0 & 0 & 0 & 0 & 0 & 0 & 0 & 0 & 0 & 0 & 0 & 0 & 0.8 & 0 & 0 & 0 & 0 \\
\hline 5 & 0 & 0 & \begin{tabular}{|l|}
0.32 \\
\end{tabular} & 0.12 & 0 & 0 & 0 & 0 & 0 & 0 & 0 & 0 & 0 & 0 & 0.72 & 0 & 0 & 0 & 0 & 0 & 0 & 0 & 0 & 0 & 0 & 0 \\
\hline 6 & 0 & 0 & 0 & 0 & 0 & 0 & 0 & 0 & 0 & 0 & 0 & 0 & 0 & 0 & 0 & 0 & 0 & 0 & 0 & 0 & 0.8 & 0 & 0 & 0 & 0 & 0 \\
\hline 7 & 0 & 0 & 0 & 0 & 0 & 0 & 0 & 0 & 0 & 0 & 0 & 0 & 0.24 & 0 & 0 & 0.44 & 0.68 & 0.92 & 0 & 0 & 0 & 0 & 0 & 0 & 0 & 0 \\
\hline 8 & 0 & 0 & 0 & 0 & 0 & 0 & 0 & 0 & 0.52 & 0 & 0 & 0 & 0 & 0 & 0 & 0 & 0 & 0.8 & 0 & 0.72 & 0 & 0 & 0 & 0 & 0 & 0 \\
\hline 9 & 0 & 0 & 0 & 0 & 0 & 0 & 0 & 0.52 & 0 & 0 & 0 & 0 & 0 & 0 & 0 & 0 & 0 & 0 & 0.44 & 0 & 0 & \begin{tabular}{|l}
0.6 \\
\end{tabular} & 0 & 0 & 0 & 0 \\
\hline 10 & 0 & 0 & 0 & 0 & 0 & 0 & 0 & 0 & 0 & 0 & 0.8 & 0 & 0 & 0 & 0 & 0 & 0 & 0 & 0 & 0 & 0 & 0.6 & 0 & 0 & 0 & 0 \\
\hline 11 & 0 & 0 & 0 & 0 & 0 & 0 & 0 & 0 & 0 & \begin{tabular}{|l|}
0.8 \\
\end{tabular} & 0 & 0 & 0 & 0 & 0 & 0 & 0 & 0 & 0 & 0 & 0 & \begin{tabular}{|l|}
0.8 \\
\end{tabular} & 0 & 0 & 0 & 0 \\
\hline 12 & 0 & 0 & 0 & 0 & 0 & 0 & 0 & 0 & 0 & 0 & 0 & 0 & 0 & 0.6 & 0 & 0 & 0 & 0 & 0 & 0 & 0 & \begin{tabular}{|l|}
0.8 \\
\end{tabular} & 0 & 0 & 0 & 0 \\
\hline 13 & 0 & 0 & 0 & 0 & 0 & 0 & 0.24 & 0 & 0 & 0 & 0 & 0 & 0 & 0 & 0 & 0 & 0 & 0 & 0 & 0 & 0 & 0 & 0 & 0 & 0 & 0 \\
\hline 14 & 0 & 0 & 0 & 0 & 0 & 0 & 0 & 0 & 0 & 0 & 0 & 0.6 & 0 & 0 & 0 & 0 & 0 & 0 & 0 & 0 & 0 & 0.32 & 0 & 0 & 0 & 0 \\
\hline 15 & 0 & 0 & 0 & 0 & 0.72 & 0 & 0 & 0 & 0 & 0 & 0 & 0 & 0 & 0 & 0 & 0 & 0.92 & 0 & 0 & 0 & 0 & 0 & 0 & 0 & 0 & 0 \\
\hline 16 & 0 & 0 & 0 & 0 & 0 & 0 & 0.44 & 0 & 0 & 0 & 0 & 0 & 0 & 0 & 0 & 0 & 0.24 & 0 & 0 & 0 & 0 & 0 & 0 & 0 & 0 & 0 \\
\hline 17 & 0 & 0 & 0 & 0 & 0 & 0 & 0.68 & 0 & 0 & 0 & 0 & 0 & 0 & 0 & 0.92 & 0.24 & 0 & 0 & 0 & 0 & 0 & 0 & 0 & 0 & 0 & 0 \\
\hline 18 & 0 & 0 & 0 & 0 & 0 & 0 & 0.92 & 0.8 & 0 & 0 & 0 & 0 & 0 & 0 & 0 & 0 & 0 & 0 & 0.8 & 0 & 0 & \begin{tabular}{|l|}
0.8 \\
\end{tabular} & 0 & 0 & 0 & 0 \\
\hline 19 & 0 & 0 & 0 & 0 & 0 & 0 & 0 & 0 & 0.44 & 0 & 0 & 0 & 0 & 0 & 0 & 0 & 0 & 0.8 & 0 & 0.8 & 0 & 0 & 0 & 0 & 0 & 0 \\
\hline 20 & 0 & 0 & 0 & 0 & 0 & 0 & 0 & 0.72 & 0 & 0 & 0 & 0 & 0 & 0 & 0 & 0 & 0 & 0 & 0.8 & 0 & 0.72 & 0 & 0 & 0 & 0 & 0 \\
\hline 21 & 0 & 0 & 0 & 0 & 0 & 0.8 & 0 & 0 & 0 & 0 & 0 & 0 & 0 & 0 & 0 & 0 & 0 & 0 & 0 & 0.72 & 0 & 0 & 0 & 0 & 0 & 0 \\
\hline 22 & 0 & 0.8 & 0 & 0.8 & 0 & 0 & 0 & 0 & 0.6 & 0.6 & 0.32 & 0.8 & 0 & 0.32 & 0 & 0 & 0 & 0 & 0 & 0 & 0 & 0 & 0.24 & 0.24 & 0 & 0.6 \\
\hline 23 & 0 & 0 & 0 & 0 & 0 & 0 & 0 & 0 & 0 & 0 & 0 & 0 & 0 & 0 & 0 & 0 & 0 & 0 & 0 & 0 & 0 & 0.24 & 0 & 0.24 & 0 & 0 \\
\hline 24 & 0.24 & 0.24 & 0 & 0 & 0 & 0 & 0 & 0 & 0 & 0 & 0 & 0 & 0 & 0 & 0 & 0 & 0 & 0 & 0 & 0 & 0 & 0.24 & 0 & 0 & 0.6 & 0 \\
\hline 25 & 0.88 & 0 & \begin{tabular}{|l|l|}
0.44 \\
\end{tabular} & 0 & 0 & 0 & 0 & 0 & 0 & 0 & 0 & 0 & 0 & 0 & 0 & 0 & 0 & 0 & 0 & 0 & 0 & 0 & 0 & 0.6 & 0 & 0 \\
\hline 26 & 0 & 0 & 0 & 0 & 0 & 0 & 0 & 0 & 0 & 0 & 0 & 0 & 0 & 0 & 0 & 0 & 0 & 0 & 0 & 0 & 0 & 0.6 & 0 & 0 & 0 & 0 \\
\hline
\end{tabular}

Fig. 9 Comprehensive correlation strength matrix I

\subsubsection{Module partition of weighted network for the differential assembly model}

In the Section 6.2.1, the comprehensive correlation strength matrix I have been evaluated. The matrix I is used as the input parameter of the FN Algorithm. The module partition results and modularity $Q$ for automobile differential assembly model as shown in Fig. 10. In the light of the modularity $Q$ in Fig. 10(a), the optimal modularity is $Q=0.5112$, and corresponding number of communities is 5 . Therefore, the desired module partition results of the gear oil pump assembly model are obtained when the number of modules is 5 , and the part nodes set $C_{1}=\left\{v_{5}, v_{15}, v_{17}\right\}, C_{2}=\left\{v_{1}, v_{2}, v_{3}, v_{4}\right.$, $\left.v_{23}, v_{24}, v_{25}\right\}, C_{3}=\left\{v_{10}, v_{11}, v_{12}, v_{14}, v_{22}, v_{26}\right\}, C_{4}=\left\{v_{6}, v_{21}\right\}$ and $C_{5}=\left\{v_{7}, v_{8}, v_{9}, v_{13}, v_{16}, v_{18}, v_{19}, v_{20}\right\}$. Furthermore, the modules partition result of automobile differential assembly model fits well with the actual situation.
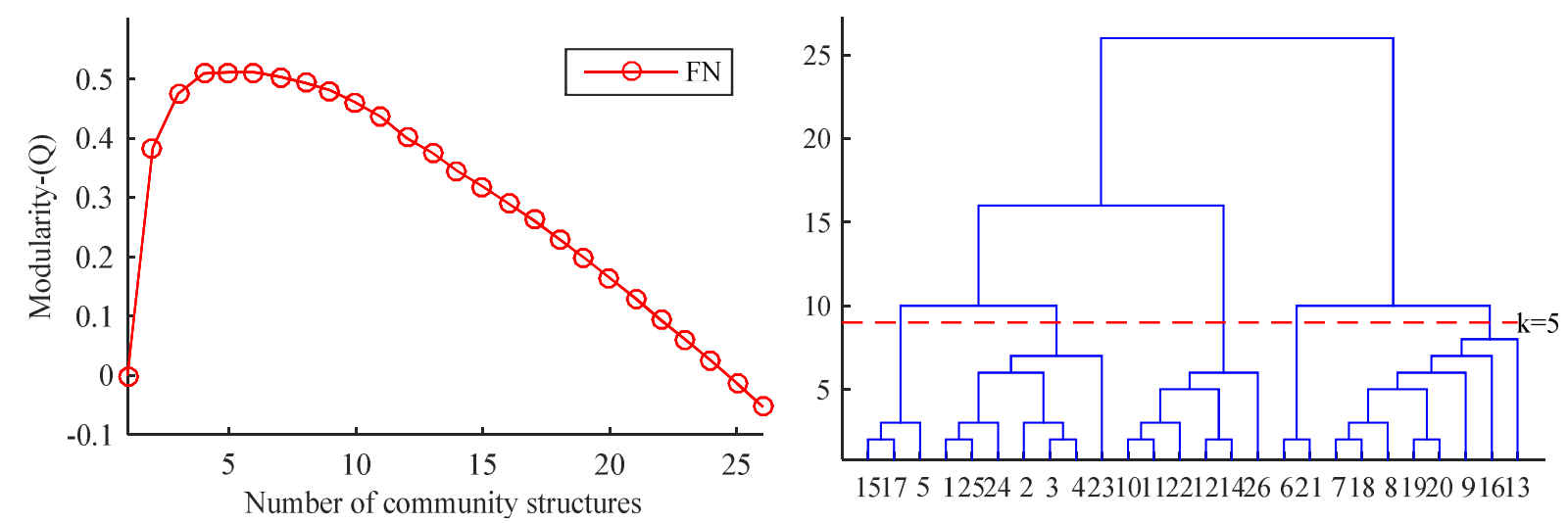

(a) The modularity $Q$ for FN

(b) The dendrogram of module partition based on FN

Fig. 10 Module partition results and modularity $Q$ for the automobile differential assembly model

The above two CAD assembly models belonging to two different categories of products are tested and divided into some modules by means of community detection. The given approach works well and easy to be implemented.

\section{Conclusion and future work}

In this paper, a novel method for module partition of mechanical CAD assembly model based on community detection is proposed. The correlation strength between assembled parts is evaluated based on their mulit-source corrlation information. Addtionally, a weighed network is used to express the correlation relationships between assembled parts. Basd on this, An improved FN algorithm is put forward to identify and discover community in weighed network corresponding to the mechanical CAD assembly model, which is simple and easy to implement. 
The proposed method is flexible and suitable for various kinds of complex mechanical assembly model by comprehensive evaluation of multi-source correlation information according to specific requirements, identifies conveniently, quickly and accurately the modular structures in CAD assembly model. It can not only help designers analyze and manage plenty of CAD assembly models created in the product development, but also assist engineers search easily the reusable assembled module, ease the difficulty in assembly retrieval and enhance the reuse level of complex CAD assembly model. Subsequent studies aim at parallel retrieval and assembled module reuse for CAD assembly model.

\section{Acknowledgements}

This work is supported by the Project Supported by Natural Science Basic Research Plan in Shaanxi Province of China (No. 2016JM5040).

\section{References}

Bonvoisin J., Halstenberg F., Buchert T. and Stark R., A systematic literature review on modular product design, Journal of Engineering Design, Vol. 27, No. 7 (2016), pp. 488-514.

Cao H., Mo R., Wan N., Shang F., Li C. and Zhang D., A subassembly identification method for truss structures manufacturing based on community detection, Vol. 35, No. 3 (2015), pp. 249-258.

Chen X., Gao S., Guo S. and Bai J., A flexible assembly retrieval approach for model reuse, Computer-Aided Design, Vol. 44, No. 6 (2012), pp. 554-574.

Deshmukh A. S., Banerjee A. G., Gupta S. K. and Sriram R. D., Content-based assembly search: A step towards assembly reuse, Computer-Aided Design, Vol. 40, No. 2 (2008), pp. 244-261.

Gao F., Xiao G. and Simpson T. W., Identifying functional modules using generalized directed graphs: Definition and application, Computers in Industry, Vol. 61, No. 3 (2010), pp. 260-269.

Girvan M. and Newman M. E., Community structure in social and biological networks, Proceedings of the national academy of sciences, Vol. 99, No. 12 (2002), pp. 7821-7826.

$\mathrm{Gu}$ P. and Sosale S., Product modularization for life cycle engineering, Robotics and Computer-Integrated Manufacturing, Vol. 15, No. 5 (1999), pp. 387-401.

Han Z., Mo R., Chang Z., Hao L. and Niu W., key assembly structure identification in complex mechanical assembly based on multi-source information, Assembly Automation, Vol. 37, No. 2 (2017), pp. 208-218.

Helmer R., Yassine A. and Meier C., Systematic module and interface definition using component design structure matrix, Journal of Engineering Design, Vol. 21, No. 6 (2010), pp. 647-675.

$\mathrm{Hu}$ K., Wang B., Yong J. and Paul J., Relaxed lightweight assembly retrieval using vector space model, Computer-Aided Design, Vol. 45, No. 3 (2013), pp. 739-750.

Huang C., Liang W., Chuang H. and Chang Z., A novel approach to product modularity and product disassembly with the consideration of 3R-abilities, Computers \& Industrial Engineering, Vol. 62, No. 1 (2012), pp. 96-107.

Kreng V. B. and Lee T., Modular product design with grouping genetic algorithm-a case study, Computers \& Industrial Engineering, Vol. 46, No. 3 (2004), pp. 443-460.

Li B. M. and Xie S. Q., Module partition for 3D CAD assembly models: a hierarchical clustering method based on component dependencies, International Journal of Production Research, Vol. 53, No. 17 (2015), pp. 5224-5240.

Li S. and Mirhosseini M., A matrix-based modularization approach for supporting secure collaboration in parametric design, Computers in Industry, Vol. 63, No. 6 (2012), pp. 619-631.

Ma L., Huang Z. and Wang Y., Automatic discovery of common design structures in CAD models, Computers \& Graphics, Vol. 34, No. 5 (2010), pp. 545-555.

Mathew A. and Rao C. S. P., A CAD system for extraction of mating features in an assembly, Assembly Automation, Vol. 30, No. 2 (2010), pp. 142-146.

Mourchid Y., El Hassouni M. and Cherifi H., An Image Segmentation Algorithm based on Community Detection, International Workshop on Complex Networks and their Applications (Springer, 2016), pp. 821-830.

Newman M. E. J., Analysis of weighted networks, Physical Review E, Vol. 70, No. 5 (2004), pp. 56131.

Newman M. E., Fast algorithm for detecting community structure in networks, Physical Review E, Vol. 69, No. 6 (2004), pp. 66133.

Newman M. E. and Girvan M., Finding and evaluating community structure in networks, Physical Review E, Vol. 69, No. 2 (2004), pp. 26113. 
Ulrich K., Fundamentals of product modularity, Management of Design (Springer, 1994), pp. 219-231.

Wang P., Li Y., Zhang J. and Yu J., An assembly retrieval approach based on shape distributions and Earth Mover's Distance, The International Journal of Advanced Manufacturing Technology, Vol. 86, No. 9-12 (2016), pp. 2635-2651.

Wang Q., Li W., Zhang X. and Lu S., Academic Paper Recommendation Based on Community Detection in Citation-Collaboration Networks, Asia-Pacific Web Conference (Springer, 2016), pp. 124-136.

Zhang J., Xu Z., Li Y., Jiang S. and Wei N., Generic face adjacency graph for automatic common design structure discovery in assembly models, Computer-Aided Design, Vol. 45, No. 8 (2013), pp. 1138-1151. 